Journal of Nonlinear Mathematical Physics

ISSN (Online): 1402-9251 ISSN (Print): 1776-0852

$A^{\text {arunatis }}$ Journal Home Page: https://www.atlantis-press.com/journals/jnmp

\title{
On the Recurrence Coefficients for Generalized $q$-Laguerre Polynomials
}

Galina Filipuk, Christophe Smet

To cite this article: Galina Filipuk, Christophe Smet (2013) On the Recurrence Coefficients for Generalized $q$-Laguerre Polynomials, Journal of Nonlinear Mathematical Physics 20: Supplement 1, 48-56, DOI: https://doi.org/10.1080/14029251.2013.862431

To link to this article: https://doi.org/10.1080/14029251.2013.862431

Published online: 04 January 2021 


\title{
On the Recurrence Coefficients for Generalized $q$-Laguerre Polynomials
}

\author{
Galina Filipuk \\ Faculty of Mathematics, Informatics and Mechanics, University of Warsaw, Banacha 2 \\ Warsaw, 02-097, Poland \\ filipuk@mimuw.edu.pl \\ Christophe Smet \\ Department of Mathematics, Katholieke Universiteit Leuven Celestijnenlaan 200B box 2400 \\ Leuven, BE-3001, Belgium \\ chr.smet@gmail.com \\ Received 31 May 2012 \\ Accepted 27 May 2013
}

\begin{abstract}
In this paper we consider a semi-classical variation of the weight related to the $q$-Laguerre polynomials and study their recurrence coefficients. In particular, we obtain a second degree second order discrete equation which in particular cases can be reduced to either the $q P_{V}$ or the $q P_{I I I}$ equation.
\end{abstract}

Keywords: Orthogonal polynomials; discrete Painlevé equations.

2000 Mathematics Subject Classification: 34M55

\section{Introduction}

\subsection{Orthogonal polynomials}

One of the most important properties of orthogonal polynomials is the three-term recurrence relation $[6,13]$. For a sequence $\left(P_{n}\right)_{n \geq 0}$ of monic polynomials (of degree $n$ in $x$ ) orthogonal with respect to a positive measure $\mu$ with support on the real line

$$
\int P_{n}(x) P_{m}(x) d \mu(x)=\zeta_{n} \delta_{n, m}, \quad \zeta_{n}>0, \quad n, m=0,1,2, \ldots,
$$

where $\delta_{n, m}$ is the Kronecker delta, this relation takes the following form:

$$
x P_{n}(x)=P_{n+1}(x)+\alpha_{n} P_{n}(x)+\beta_{n} P_{n-1}(x)
$$

with the recurrence coefficients given by the following integrals

$$
\alpha_{n}=\frac{1}{\zeta_{n}} \int x P_{n}^{2}(x) d \mu(x), \quad \beta_{n}=\frac{1}{\zeta_{n-1}} \int x P_{n}(x) P_{n-1}(x) d \mu(x) .
$$

The initial conditions are taken to be $\beta_{0} P_{-1}=0$ and $P_{0}=1$. In this paper we use the following expression for monic polynomials:

$$
P_{n}(x)=x^{n}+\gamma_{n} x^{n-1}+\ldots
$$


In this paper we will work with a weight function $w$ on the positive half line, so that the orthogonality conditions can be written as

$$
\int_{0}^{\infty} P_{n}(x) P_{m}(x) w(x) d x=\zeta_{n} \delta_{n, m}
$$

The recurrence coefficients can be expressed in terms of determinants containing the moments of the orthogonality measure [6]. For classical orthogonal polynomials (Hermite, Laguerre, Jacobi) one knows these recurrence coefficients explicitly in contrast to non-classical weights. The recurrence coefficients of semi-classical weights obey nonlinear recurrence relations, which, in many cases, can be identified as discrete Painlevé equations; see [4] and the references therein. The Painlevé equations (both discrete and continuous) have many important applications in mathematics and mathematical physics; cf. [7, 12,16]. Relations between the semi-classical orthogonal polynomials and the continuous Painlevé equations can be found in, for instance, [2,9,11,15]. Relations between the semi-classical orthogonal polynomials and the discrete Painlevé equations can be found in, for instance, $[1,4,14,17]$ and the references therein.

In this paper we consider the recurrence coefficients of the generalized $q$-Laguerre polynomials for the weight function

$$
w(x)=\frac{x^{\alpha}\left(-p_{1} / x ; q\right)_{\infty}\left(-p_{2} / x ; q\right)_{\infty}}{\left(-x^{2} ; q^{2}\right)_{\infty}\left(-q^{2} / x^{2} ; q^{2}\right)_{\infty}}
$$

where $x \in(0,+\infty),|q|<1, p_{1}>0, p_{2}>0, p_{1} p_{2}<q^{2-\alpha}, \alpha \geq 0$. Here

$$
(a ; q)_{\infty}=\prod_{k=0}^{\infty}\left(1-a q^{k}\right) .
$$

The case $p_{1}+p_{2}=0, p_{1} p_{2}=-p$ was considered in Section 7.2 [1]. It was shown that the recurrence coefficients are related to the $q$-discrete Painlevé equation $q P_{V}$. A particular case when $p=q^{2}$ was considered in [4]. The proof was based on the compatibility of the ladder operators for orthogonal polynomials [5].

\subsection{Ladder operators}

In the case of continuous $q$-orthogonal polynomials on the positive half line, the ladder operators were first considered in [5]. We repeat the main statements which we use later on to be selfcontained following [5] and Section 1.3 [1].

The $q$-difference operator is given by

$$
\left(D_{q} f\right)(x)= \begin{cases}\frac{f(x)-f(q x)}{x(1-q)}, & x \neq 0, \\ f^{\prime}(0) & x=0 .\end{cases}
$$

Let us define the function $u$, called the potential, by the following formula:

$$
u(x)=-\frac{D_{q^{-1}} w(x)}{w(x)} .
$$

Then the polynomials satisfy the following lowering equation:

$$
D_{q} P_{n}(x)=A_{n}(x) P_{n-1}(x)-B_{n}(x) P_{n}(x),
$$


where the functions $A_{n}(x)$ and $B_{n}(x)$ are given by

$$
\begin{gathered}
A_{n}(x)=\frac{1}{\zeta_{n}} \int_{0}^{\infty} \frac{u(q x)-u(y)}{q x-y} P_{n}(y) P_{n}(y / q) w(y) d y, \\
B_{n}(x)=\frac{1}{\zeta_{n-1}} \int_{0}^{\infty} \frac{u(q x)-u(y)}{q x-y} P_{n}(y) P_{n-1}(y / q) w(y) d y .
\end{gathered}
$$

Furthermore, the following relations (compatibility conditions) hold:

$$
\begin{gathered}
B_{n+1}(x)+B_{n}(x)=\left(x-\alpha_{n}\right) A_{n}(x)+x(q-1) \sum_{j=0}^{n} A_{j}(x)-u(q x), \\
1+\left(x-\alpha_{n}\right) B_{n+1}(x)-\left(q x-\alpha_{n}\right) B_{n}(x)=\beta_{n+1} A_{n+1}(x)-\beta_{n} A_{n-1}(x) .
\end{gathered}
$$

In addition, we have the following identities:

$$
\int_{0}^{\infty} u(y) P_{n}(y) P_{n}(y / q) w(y) d y=0
$$

and

$$
\int_{0}^{\infty} u(y) P_{n+1}(y) P_{n}(y / q) w(y) d y=\frac{1-q^{n+1}}{1-q} q \zeta_{n}
$$

They are due to orthogonality and the integration by parts formula

$$
\int_{0}^{\infty} f(x) D_{q} g(x) d x=-\frac{1}{q} \int_{0}^{\infty} g(x) D_{q^{-1}} f(x) d x,
$$

where the good behaviour of the weight near zero and infinity guarantees that this formula can be applied.

\section{Main Result}

In this section we prove the main result for the recurrence coefficients of monic orthogonal polynomials with respect to the weight (1.6).

Theorem 2.1. The recurrence coefficients $\alpha_{n}$ and $\beta_{n}$ in the three-term recurrence relation for monic polynomials

$$
x P_{n}(x)=P_{n+1}(x)+\alpha_{n} P_{n}(x)+\beta_{n} P_{n-1}(x)
$$

for the weight

$$
w(x)=\frac{x^{\alpha}\left(-p_{1} / x ; q\right)_{\infty}\left(-p_{2} / x ; q\right)_{\infty}}{\left(-x^{2} ; q^{2}\right)_{\infty}\left(-q^{2} / x^{2} ; q^{2}\right)_{\infty}}
$$

with $x \in(0,+\infty),|q|<1, p_{1}>0, p_{2}>0, p_{1} p_{2}<q^{2-\alpha}, \alpha \geq 0$, can be expressed in terms of the function $y_{n}$, which satisfies the second order second degree discrete equation

$$
\left(c_{n}^{2}-b_{n} b_{n-1}\right)^{2}-a_{n} a_{n-1} c_{n}\left(c_{n}^{2}+b_{n} b_{n-1}\right)-c_{n}^{2}\left(a_{n}^{2} b_{n-1}+a_{n-1}^{2} b_{n}\right)=0,
$$


with

$$
\begin{aligned}
& a_{n}=q^{n-1}\left(p_{1}+p_{2}\right), \\
& b_{n}=q^{2 n+\alpha}\left(y_{n+1} y_{n}-p_{1} p_{2} q^{-\alpha-2}\right), \\
& c_{n}=q^{n-1} \frac{\left(y_{n}+q^{-\alpha}\right)\left(y_{n}+p_{1} p_{2} q^{-2}\right)}{y_{n}+q^{-n-\alpha}},
\end{aligned}
$$

where

$$
\beta_{n}=q^{1-n}\left(y_{n}+q^{-n-\alpha}\right)
$$

and

$$
\begin{aligned}
q^{2 \alpha+2 n+2}\left(q^{\alpha+2} y_{n} y_{n+1}-\right. & \left.p_{1} p_{2}\right) \alpha_{n}^{2}+\left(p_{1}+p_{2}\right) q^{\alpha+n+1}\left(q^{2}+q^{\alpha}\left(p_{1} p_{2}+q^{2}\left(y_{n}+y_{n+1}\right)\right)\right) \alpha_{n} \\
& +\left(q^{2}+q^{\alpha}\left(p_{1} p_{2}+q^{2}\left(y_{n}+y_{n+1}\right)\right)\right)^{2}=0 .
\end{aligned}
$$

Proof. The method applied in the proof is based on the method used in Th. 5.3.2 [1] and earlier in [4].

Using the definition of the weight function, it is easy to find the following relation:

$$
w(x / q)=\frac{q^{2-\alpha}}{\left(x+p_{1}\right)\left(x+p_{2}\right)} w(x) .
$$

The potential (1.7) is given by

$$
u(x)=\frac{q}{1-q} \frac{1}{x}-\frac{q^{3-\alpha}}{1-q} \frac{1}{x\left(x+p_{1}\right)\left(x+p_{2}\right)} .
$$

Next one can compute

$$
\frac{u(q x)-u(y)}{q x-y}=-\frac{1}{q x} u(y)+\frac{q^{2-\alpha}}{1-q} \frac{1}{\left(y+p_{1}\right)\left(y+p_{2}\right)}\left(\frac{q}{\left(q x+p_{1}\right)\left(q x+p_{2}\right)}+\frac{p_{1}+p_{2}+y}{x\left(q x+p_{1}\right)\left(q x+p_{2}\right)}\right) .
$$

The functions $A_{n}(x)$ and $B_{n}(x)$ in, respectively, Eq. (1.8) and Eq. (1.9) can be found as follows. We have

$$
A_{n}(x)=\frac{q^{2}}{1-q} \frac{T_{n}}{x\left(q x+p_{1}\right)\left(q x+p_{2}\right)}+\frac{q^{n+2}}{1-q} \frac{1}{\left(q x+p_{1}\right)\left(q x+p_{2}\right)}
$$

where

$$
T_{n}=q^{n-1}\left(p_{1}+p_{2}+\gamma_{n}-q \gamma_{n+1}\right)
$$

since $P_{n}(q u) u=q^{n} P_{n+1}(u)+q^{n-1}\left(\gamma_{n}-q \gamma_{n+1}\right) P_{n}(u)+$ lower order terms. Thus,

$$
\sum_{j=0}^{n} T_{j}=\frac{\left(p_{1}+p_{2}\right)}{q} \frac{1-q^{n+1}}{1-q}-q^{n} \gamma_{n+1} .
$$

Similarly,

$$
B_{n}(x)=-\frac{1}{x} \frac{1-q^{n}}{1-q}+\frac{q^{2}}{1-q} \frac{r_{n}}{\left(q x+p_{1}\right)\left(q x+p_{2}\right)}+\frac{q^{2}}{1-q} \frac{t_{n}}{x\left(q x+p_{1}\right)\left(q x+p_{2}\right)}
$$


with

$$
\begin{gathered}
r_{n}=\frac{1}{\zeta_{n-1}} \int_{0}^{\infty} P_{n}(q u) P_{n-1}(u) w(u) d u \\
t_{n}=\frac{p_{1}+p_{2}}{q \zeta_{n-1}} \int_{0}^{\infty} P_{n}(q u) P_{n-1}(u) w(u) d u+\frac{1}{\zeta_{n-1}} \int_{0}^{\infty} u P_{n}(q u) P_{n-1}(u) w(u) d u .
\end{gathered}
$$

Clearly, $r_{0}=t_{0}=0$. One can further calculate $r_{n}$. Indeed, since $P_{n}(q u)=q^{n} P_{n}(u)+(1-$ q) $\gamma_{n} q^{n-1} P_{n-1}(u)+$ lower order terms, and using orthogonality we have

$$
r_{n}=(1-q) \gamma_{n} q^{n-1} .
$$

The compatibility conditions (1.10) and (1.11) give the following relations:

$$
\begin{gathered}
t_{n+1}+t_{n}+p_{1} p_{2} q^{-2}\left(q^{n+1}+q^{n}-1\right)=q^{-\alpha}-\alpha_{n} T_{n}, \\
r_{n+1}+r_{n}=-\alpha_{n} q^{n}+T_{n}-(1-q) \sum_{j=0}^{n} T_{j}-\left(q^{n}+q^{n-1}-q^{-1}\right)\left(p_{1}+p_{2}\right), \\
r_{n+1}-q r_{n}+q^{n}(1-q) \alpha_{n}=0, \\
\beta_{n+1} T_{n+1}-\beta_{n} T_{n-1}=-\alpha_{n}\left(t_{n+1}-t_{n}\right)+\alpha_{n} q^{n}(1-q) p_{1} p_{2} q^{-2}, \\
t_{n+1}-q t_{n}-\alpha_{n} r_{n+1}+\alpha_{n} r^{n}=\beta_{n+1} q^{n+1}-\beta_{n} q^{n-1}-q^{n-1}(1-q)\left(p_{1}+p_{2}\right) \alpha_{n} .
\end{gathered}
$$

Using Eq. (2.1) and the fact that $\alpha_{n}=\gamma_{n}-\gamma_{n+1}$ which can be easily seen from the three-term recurrence relation comparing the coefficients at $x^{n}$, Eq. (2.6) is trivial (i.e., the equation is automatically satisfied). Similarly, using Eq. (2.1), Eq. (2.3) and Eq. (2.2), Eq. (2.5) is also trivial. Further, using Eq. (2.1) and Eq. (2.3) we have

$$
r_{n+1}-r_{n}=(1-q)\left(\gamma_{n+1} q^{n}-\gamma_{n} q^{n-1}\right)=(1-q)\left(\left(p_{1}+p_{2}\right) q^{n-1}-T_{n}\right) .
$$

Inserting this into Eq. (2.8) we obtain

$$
t_{n+1}-q t_{n}+(1-q) \alpha_{n} T_{n}=\beta_{n+1} q^{n+1}-\beta_{n} q^{n-1} .
$$

Using Eq. (2.4) for replacement of $\alpha_{n} T_{n}$ in the last expression and multiplying both sides by $q^{n}$ we obtain

$$
q^{n-\alpha}(1-q)+q^{n+1} t_{n+1}-q^{n} t_{n}+(1-q) q^{-2} p_{1} p_{2}\left(q^{n}-q^{2 n}(1+q)\right)=\beta_{n+1} q^{2 n+1}-\beta_{n} q^{2 n-1} .
$$

Taking a telescopic sum (i.e., summing the last expression from 0 to $n-1$ ), we get

$$
\beta_{n} q^{2 n-1}=\left(1-q^{n}\right)\left(q^{-\alpha}+p_{1} p_{2} q^{-2}\right)-\left(1-q^{2 n}\right) p_{1} p_{2} q^{-2}+q^{n} t_{n} .
$$

Thus, $\beta_{n}$ is expressed in terms of $t_{n}$. 
Next, we multiply Eq. (2.7) by $T_{n}$. The expression $\alpha_{n} T_{n}$ is replaced by using Eq. (2.4) for the term $t_{n+1}-t_{n}$ and by using Eq. (2.9) in another case. We also use Eq. (2.10) on the right hand side to replace $\beta_{n}$. As a result we have

$$
\begin{gathered}
\beta_{n+1} T_{n+1} T_{n}-\beta_{n} T_{n} T_{n-1}=t_{n+1}^{2}-t_{n}^{2}-\left(t_{n+1}-t_{n}\right)\left(q^{-\alpha}+p_{1} p_{2} q^{-2}\right)+2 p_{1} p_{2} q^{n-1} t_{n+1} \\
-2 p_{1} p_{2} q^{n-2} t_{n}+q^{n-2}(1-q)\left(q^{-\alpha}+p_{1} p_{2} q^{-2}\right) p_{1} p_{2}-q^{2 n-4}\left(1-q^{2}\right) p_{1}^{2} p_{2}^{2} .
\end{gathered}
$$

Taking the telescopic sum and taking into account that $t_{0}=\beta_{0}=0$, we get

$$
\begin{aligned}
\beta_{n} T_{n} T_{n-1}= & t_{n}^{2}-t_{n}\left(q^{-\alpha}+p_{1} p_{2} q^{-2}\right)+2 p_{1} p_{2} q^{n-2} t_{n} \\
& +\left(1-q^{n}\right)\left(q^{-\alpha}+p_{1} p_{2} q^{-2}\right) p_{1} p_{2} q^{-2}-\left(1-q^{2 n}\right) p_{1}^{2} p_{2}^{2} q^{-4}
\end{aligned}
$$

Replacing $t_{n}$ and $t_{n+1}$ in Eq. (2.7) using Eq. (2.10) yields

$$
\beta_{n+1}\left(T_{n+1}+q^{n} \alpha_{n}\right)-\beta_{n}\left(T_{n-1}+q^{n-1} \alpha_{n}\right)=\alpha_{n}(1-q) q^{-n-\alpha-1} .
$$

Replacing $\alpha_{n}=\gamma_{n}-\gamma_{n+1}$ and $T_{n}$ by Eq. (2.1) on the left hand side, we get

$$
\begin{gathered}
\beta_{n+1}\left(p_{1}+p_{2}+\gamma_{n}-q \gamma_{n+2}\right) q^{2 n+1} \\
-\beta_{n}\left(p_{1}+p_{2}+\gamma_{n-1}-q \gamma_{n+1}\right) q^{2 n-1}=\alpha_{n}(1-q) q^{-\alpha} .
\end{gathered}
$$

Taking a telescopic sum, we obtain

$$
q^{2 n+\alpha-1} \beta_{n}\left(p_{1}+p_{2}+\gamma_{n-1}-q \gamma_{n+1}\right)=(q-1) \gamma_{n} .
$$

Using Eq. (2.1) and replacing $\gamma_{n+1}=\gamma_{n}-\alpha_{n}$ we get

$$
q^{n} \alpha_{n}=T_{n}-q^{n-1}\left(p_{1}+p_{2}+(1-q) \gamma_{n}\right) .
$$

Next we replace $\gamma_{n}$ using Eq. (2.12) and again use $\gamma_{n+1}=\gamma_{n}-\alpha_{n}$. We also express $\gamma_{n}$ in terms of $T_{n-1}$ and $\gamma_{n-1}$ from Eq. (2.1). Finally we get

$$
\alpha_{n} q^{n}\left(1-q^{2 n+\alpha-1} \beta_{n}\right)=T_{n}+q^{2 n+\alpha} \beta_{n} T_{n-1}-q^{n-1}\left(p_{1}+p_{2}\right) .
$$

Next we multiply Eq. (2.13) by $T_{n}$ and substitute $\alpha_{n} T_{n}$ into Eq. (2.4). As a result we get

$$
\begin{gathered}
q^{n}\left(1-q^{2 n+\alpha-1} \beta_{n}\right)\left(q^{-\alpha}-t_{n+1}-t_{n}-\left(q^{n+1}+q^{n}-1\right) p_{1} p_{2} q^{-2}\right)= \\
T_{n}^{2}+q^{2 n+\alpha} \beta_{n} T_{n} T_{n-1}-q^{n-1}\left(p_{1}+p_{2}\right) T_{n} .
\end{gathered}
$$

In the last expression we use Eq. (2.11) to replace $\beta_{n} T_{n} T_{n-1}$ and Eq. (2.10) to replace $\beta_{n}$. We also introduce a new variable

$$
y_{n}=t_{n}-q^{-\alpha}-\left(1-q^{n}\right) p_{1} p_{2} q^{-2} .
$$

Finally, we get

$$
T_{n}\left(T_{n}-q^{n-1}\left(p_{1}+p_{2}\right)\right)=q^{2 n+\alpha}\left(y_{n+1} y_{n}-p_{1} p_{2} q^{-\alpha-2}\right) .
$$

Eq. (2.11) can be written in new variables as

$$
q^{1-n}\left(y_{n}+q^{-n-\alpha}\right) T_{n} T_{n-1}=\left(y_{n}+q^{-\alpha}\right)\left(y_{n}+p_{1} p_{2} q^{-2}\right)
$$


since from Eq. (2.10) we have

$$
\beta_{n}=q^{1-n}\left(y_{n}+q^{-n-\alpha}\right) .
$$

Eq. (2.14) and Eq. (2.15) can be used to eliminate $T_{n}$ and $T_{n-1}$ to get a second order second degree nonlinear discrete equation for $y_{n}$ (here we mean that the equation involves $y_{n-1}, y_{n}, y_{n+1}$ and is of second degree in $y_{n+1}$ ) as follows: to eliminate $T_{n}$ one can compute the resultant for Eq. (2.14) and Eq. (2.15). If we first rewrite these two equations as

$$
\begin{array}{r}
T_{n}^{2}-a_{n} T_{n}-b_{n}=0, \\
T_{n-1} T_{n}-c_{n}=0,
\end{array}
$$

with

$$
\begin{aligned}
a_{n} & =q^{n-1}\left(p_{1}+p_{2}\right), \\
b_{n} & =q^{2 n+\alpha}\left(y_{n+1} y_{n}-p_{1} p_{2} q^{-\alpha-2}\right), \\
c_{n} & =q^{n-1} \frac{\left(y_{n}+q^{-\alpha}\right)\left(y_{n}+p_{1} p_{2} q^{-2}\right)}{y_{n}+q^{-n-\alpha}}
\end{aligned}
$$

this leads to

$$
b_{n} T_{n-1}^{2}+a_{n} c_{n} T_{n-1}-c_{n}^{2}=0 .
$$

Next, using this expression and Eq. (2.17) with $n-1$, one eliminates $T_{n-1}$, again by computing the resultant. This gives

$$
\left(c_{n}^{2}-b_{n} b_{n-1}\right)^{2}-a_{n} a_{n-1} c_{n}\left(c_{n}^{2}+b_{n} b_{n-1}\right)-c_{n}^{2}\left(a_{n}^{2} b_{n-1}+a_{n-1}^{2} b_{n}\right)=0 .
$$

Substituting the expression of $\beta_{n}$ in terms of $y_{n}$ into Eq. (2.13) and replacing $T_{n-1}$ from Eq. (2.15), we get an expression for $\alpha_{n}, y_{n}$ and $T_{n}$. Eliminating $T_{n}$ between this expression and Eq. (2.14), we get a quadratic expression for $\alpha_{n}$ in terms of $y_{n}$ and $y_{n+1}$ :

$$
\begin{aligned}
q^{2 \alpha+2 n+2}\left(q^{\alpha+2} y_{n} y_{n+1}-\right. & \left.p_{1} p_{2}\right) \alpha_{n}^{2}+\left(p_{1}+p_{2}\right) q^{\alpha+n+1}\left(q^{2}+q^{\alpha}\left(p_{1} p_{2}+q^{2}\left(y_{n}+y_{n+1}\right)\right)\right) \alpha_{n} \\
& +\left(q^{2}+q^{\alpha}\left(p_{1} p_{2}+q^{2}\left(y_{n}+y_{n+1}\right)\right)\right)^{2}=0 .
\end{aligned}
$$

This completes the proof of the theorem.

In particular, formally if $p_{1}+p_{2}=0$ and $p=-p_{1}^{2}$, all $a_{i}$ are zero and we obtain $c_{n}^{2}=b_{n} b_{n-1}$, or in terms of $y_{n}$ :

$$
\left(y_{n} y_{n-1}-p q^{-\alpha-2}\right)\left(y_{n} y_{n+1}-p q^{-\alpha-2}\right)=\frac{\left(y_{n}+q^{-\alpha}\right)^{2}\left(y_{n}+p q^{-2}\right)^{2}}{\left(q^{\alpha+n} y_{n}+1\right)^{2}} .
$$

This case was considered in Section 7.2 [1] and was shown to be a particular case of $q P_{V}$ after some change of variables. If we take $p_{1}=p_{2}=0$, i.e. a special case of the previous one, we get the equation

$$
y_{n-1} y_{n+1}=\frac{\left(y_{n}+q^{-\alpha}\right)^{2}}{\left(q^{n+\alpha} y_{n}+1\right)^{2}} .
$$

This is the $q$-discrete Painlevé equation $q P_{I I I}$, obtained in Section 7.1 [1]. 
The initial conditions for this recurrence relation can be expressed in terms of the moments of the weight. With

$$
\mu_{k}=\int_{0}^{\infty} x^{k} w(x) d x
$$

we have from Eq. (2.16) and $\beta_{0}=0$ that $y_{0}=-q^{-\alpha}$, and from Eq. (2.21) and $\alpha_{0}=\frac{\mu_{1}}{\mu_{0}}$ we deduce that

$$
y_{1}=-\frac{\mu_{1}^{2}}{\mu_{0}^{2}}-\frac{p_{1}+p_{2}}{q} \frac{\mu_{1}}{\mu_{0}}-\frac{p_{1} p_{2}}{q^{2}} .
$$

However, it should be noted that the recurrence relation (2.20) is quadratic in $y_{n+1}$, so when one tries to find $y_{n+1}$ out of $y_{n-1}$ and $y_{n}$, one still has a sign choice to make. It is clear that $\beta_{n}>0$ for $n>0$, but this additional information does not seem to allow one to pick the right sign. This is a complication that was not present in [1]. A similar remark holds when one tries to find $\alpha_{n}$ from $y_{n}$, using Eq. (2.21). Moreover, the method of using a Painlevé equation to calculate recurrence coefficients is usually an unstable one, see e.g. Sections 3.3 and 6.5 in [1], or [3].

Remark 2.1. ${ }^{\text {a }}$ Eq. (2.20) can be further simplified and written as follows:

$$
\left(c_{n}^{2}-b_{n} b_{n-1}\right)^{2}=c_{n}\left(a_{n-1} c_{n}+a_{n} b_{n-1}\right)\left(a_{n} c_{n}+a_{n-1} b_{n}\right) .
$$

By setting

$$
a=p_{1}+p_{2}, B_{n}=q^{n+\alpha}\left(y_{n+1} y_{n}-p_{1} p_{2} q^{-\alpha-2}\right), C_{n}=\frac{\left(y_{n}+q^{-\alpha}\right)\left(y_{n}+p_{1} p_{2} q^{-2}\right)}{y_{n}+q^{-n-\alpha}},
$$

Eq. (2.20) simplifies further to

$$
\left(C_{n}^{2}-q B_{n} B_{n-1}\right)^{2}=a^{2} q^{n-2} C_{n}\left(C_{n}+q B_{n-1}\right)\left(C_{n}+B_{n}\right) .
$$

However, the expressions of $\alpha_{n}$ and $\beta_{n}$ in terms of $B_{n}$ and $C_{n}$ are cumbersome.

\section{Discussions}

There are a lot of examples which show a connection between the recurrence coefficients of the orthogonal polynomials and the Painlevé equations, both discrete and continuous. A natural question arises what happens if one modifies further such weights by introducing more parameters. In this paper we studied the case of weight (1.6) and show that the recurrence coefficients satisfy a complicated second order second degree discrete equation, which in particular cases can be reduced to $q P_{V}$ or $q P_{I I I}$. In a recent preprint [8] we showed that one can simultaneously consider a large class of weights giving potential (1.7) of a certain form and derive second order second degree discrete equations for the recurrence coefficients.

A lot of other instances of orthogonal polynomials giving rise to discrete or $q$-discrete Painlevé equations are known. We give a few examples of the weight, the corresponding Painlevé equation and a reference.

- $w(x)=|x|^{\rho} e^{-x^{4}}$ on $\mathbb{R}$ is related to $d P_{I}[10,11,14] ;$

$\overline{{ }^{a}}$ The authors are grateful to the referee for suggesting the expressions in this remark. 
- $w(k)=\frac{a^{k}}{(k !)^{2}}$ on $\mathbb{N}$ is related to $d P_{I I}[18]$

- $w(x)=x^{\alpha} e^{-x^{2}}$ on $\mathbb{R}^{+}$is related to $d P_{I V}[4]$

- $w(x)=\left(q^{4} x^{4} ; q^{4}\right)_{\infty}$ on $\left\{ \pm q^{k} \mid k \in \mathbb{N}\right\}$ is related to $q P_{I}[17]$

- $w(x)=|x|^{\alpha}\left(q^{2} x^{2} ; q^{2}\right)_{\infty}\left(c q^{2} x^{2} ; q^{2}\right)_{\infty}$ on $\left\{ \pm q^{k} \mid k \in \mathbb{N}\right\}$ is related to $\alpha q P_{V}$ [1].

\section{Acknowledgments}

GF is supported by MNiSzW Iuventus Plus Grant Nr 0124/IP3/2011/71. Part of this work was done when CS was visiting GF in Warsaw, and he is grateful for the hospitality received there.

\section{References}

[1] L. Boelen, Discrete Painlevé Equations and Orthogonal Polynomials, Ph.D. thesis, Department of Mathematics K.U.Leuven (Leuven, Belgium, 2010).

[2] L. Boelen, G. Filipuk and W. Van Assche, Recurrence coefficients of generalized Meixner polynomials and Painlevé equations, J. Phys. A: Math. Theor. 44 (2011) 035202.

[3] L. Boelen, C. Smet and W. Van Assche, $q$-Discrete Painlevé equations for recurrence coefficients of modified $q$-Freud orthogonal polynomials, J. Differ. Equ. Appl. 16 (2010) 37-53.

[4] L. Boelen and W. Van Assche, Discrete Painlevé equations for recurrence coefficients of semiclassical Laguerre polynomials, Proc. Amer. Math. Soc. 138 (2010) 1317-1331.

[5] Y. Chen and M.E.H. Ismail, Ladder operators for $q$-orthogonal polynomials, J. Math. Anal. Appl. 345 (2008) $1-10$.

[6] T. S. Chihara, An Introduction to Orthogonal Polynomials (Gordon and Breach, New York, 1978).

[7] P. A. Clarkson, Painlevé equations_-nonlinear special functions, in Orthogonal Polynomials and Special Functions: Computation and Applications, Lect. Notes in Math. 1883 (Springer, Berlin, 2006), pp. 331-411.

[8] G. Filipuk and C. Smet, On the recurrence coefficients of generalized little $q$-Laguerre polynomials, arXiv:1302.1038 [math.CA], submitted.

[9] G. Filipuk and W. Van Assche, Recurrence coefficients of generalized Charlier polynomials and the fifth Painlevé equation, Proc. Amer. Math. Soc. 141 (2013) 551-562.

[10] A.S. Fokas, A.R. Its and A.V. Kitaev, Discrete Painlevé equations and their appearance in quantum gravity, Commun. Math. Phys. 42 (1991) 313-344.

[11] G. Freud, On the coefficients in the recursion formulae of orthogonal polynomials, Proc. Roy. Irish Acad. Sect. A 76 (1976) 1-6.

[12] V. I. Gromak, I. Laine and S. Shimomura, Painlevé Differential Equations in the Complex Plane, Studies in Mathematics 28 (de Gruyter, Berlin, NewYork, 2002).

[13] M. E. H. Ismail, Classical and Quantum Orthogonal Polynomials in One Variable, Encyclopedia of Mathematics and its Applications 98 (Cambridge University Press, 2005).

[14] A. P. Magnus, Freud's equations for orthogonal polynomials as discrete Painlevé equations, in Symmetries and Integrability of Difference Equations, London Math. Soc. Lecture Note Ser. 255 (Cambridge University Press, 1999), pp. 228-243.

[15] A. P. Magnus, Painlevé type differential equations for the recurrence coefficients of semi-classical orthogonal polynomials, J. Comput. Appl. Math. 57 (1995) 215-237.

[16] M. Noumi, Painlevé Equations Through Symmetry, Transl. of Math. Monographs 223 (American Mathematical Society, Providence, RI, 2004).

[17] W. Van Assche, Discrete Painlevé equations for recurrence coefficients of orthogonal polynomials, in Discrete Equations, Special Functions and Orthogonal Polynomials (World Scientific, 2007), pp. 687725.

[18] W. Van Assche and M. Foupouagnigni, Analysis of non-linear recurrence relations for the recurrence coefficients of generalized Charlier polynomials, J. Nonlinear Math. Phys. 10 Supplement 2, (2003) 231-237. 